\title{
AIAA
}

\section{MAXIMIZED GUST LOADS OF A CLOSED-LOOP, NONLINEAR AEROELASTIC SYSTEM USING NONLINEAR SYSTEMS THEORY}

\author{
Walter A. Silva \\ Aeroelasticity Branch \\ NASA Langley Research Center \\ Hampton
}

Presented at the AIAA/ASE/ASCE/AHS/ASC

40th Structures, Structural Dynamics, and Materials Conference

April 12-15, 1999

St. Louis, Missouri

AIAA Paper No. 99-1474 


\title{
MAXIMIZED GUST LOADS OF A CLOSED-LOOP, NONLINEAR AEROELASTIC SYSTEM USING NONLINEAR SYSTEMS THEORY
}

\author{
Walter A. Silva*, Boyd Perry, III** \\ Aeroelasticity Branch \\ NASA Langley Research Center
}

\begin{abstract}
The problem of computing the maximized gust load for a nonlinear, closed-loop aeroelastic aircraft is discussed. The Volterra theory of nonlinear systems is applied in order to define a linearized system that provides a bounds on the response of the nonlinear system of interest. The method is applied to a simplified model of an Airbus A310.
\end{abstract}

\section{INTRODUCTION}

The design of an aircraft structure requires an evaluation of the loads that the aircraft is expected to encounter during its life cycle. These include taxi, landing, maneuver, and gust loads. Aircraft manufacturers have developed methods that are suitable for certification of the aircraft by the U.S. Federal Aviation Administration (FAA). These methods are based on linear principles and are, therefore, limited to linear mathematical models of the aircraft. For the majority of situations, these linear assumptions are valid and comply with certification requirements'. Quite often, however, additional improvements are made to the aircraft to further extend its life cycle such as maneuver load alleviation (MLA) and gust load alleviation (GLA) systems. These control systems transform the mathematical model of the aircraft from an open-loop (without any MLA or GLA) linear system to

* Research Scientist, Senior Member

**Assisstant Branch Head, Senior Member Copyright $\odot 1999$ by the American Institute of Aeronautics and Astronautics, Inc. No copyright is asserted in the United States under Title 17, U.S. Code. The U.S. Government has a royalty-free license to exercise all rights under the copyright claimed herein for Government Purposes. All other rights are reserved by the copyright owner. a closed-loop (with MLA or GLA) linear or nonlinear system depending on the nature of the MLA or GLA design?'

Computation of maximized, timecorrelated gust loads for a linear aeroelastic aircraft can be performed via the linear matched filter theory (MFT) approach ${ }^{3}$. The attractiveness of the MFT technique is its computational efficiency as well as the mathematical guarantees of maximality that it provides. For nonlinear, closed-loop aeroelastic aircraft, the linear MFT technique is not directly applicable, although methods have been investigated to address this issue ${ }^{4.5}$. These methods, which typically involve an optimal search of some kind, can be computationally intensive and, unfortunately, do not provide the mathematical guarantees that the linear MFT technique offers.

The goal of this paper is to present a new technique that introduces and applies nonlinear system theoretic concepts to the problem of predicting maximized gust loads for a closed-loop, nonlinear, aeroelastic aircraft. The contributions presented herein include a generalization of the linear MFT technique to nonlinear systems, a proper classification of the nonlinear nature of the problem, and results for a simplified mathematical model of a nonlinear, closedloop aeroelastic aircraft.

The paper begins with a description of the problem including a generic block diagram of the system under investigation. The nonlinear nature of the system is dicussed in order to define appropriate analyses techniques. Methodologies are then introduced for linear (Matched Filter Theory, MFT) and nonlinear (Volterra theory) systems. The relationship between the Volterra theory of nonlinear systems and MFT is presented and a strategy for defining a bounded (maximized) function for gust loads is discussed. Results are presented for a simplified aeroelastic state-space model of 
an Airbus $\mathrm{A} 310^{\circ}$ with a nonlinear gust load alleviation system.

\section{PROBLEM DESCRIPTION}

The selection of a linear or nonlinear gust loads analysis technique depends on the nature of the system being investigated. If the system consists of an open-loop, linear state-space model of an aeroelastic vehicle, then linear methods are applied. If the system consists of a linear state-space model of an aeroelastic vehicle with a linear controller closing the feedback loop, then, once again, linear methods are applied. If, however, a nonlinear controller replaces the linear controller in the closed-loop system, then nonlinear analysis methods must be employed. There are additional configurations that include a nonlinear aeroelastic system, but that class of configurations is beyond the scope of this paper.

If the GLA system includes nonlinear elements such as saturation or deadband functions, then the GLA system is nonlinear. However, since an actual GLA system can be quite complex, a simplified model has been established that can be used for developing methods of a fundamental nature $^{6}$. Figure 1 is a schematic of the general structure of the simplified model which replaces a GLA system with a simple nonlinear element. It should be restated that the purpose of this simplification is to aid in the development of new techniques and is not meant to be representative of a realistic GLA system. Details of a simplified Airbus A310 aeroelastic model used in the present research are presented in a subsequent section.

The method presented in this paper (subsequently referred to as the indirect method) consists of a combination of concepts and methodologies. The primary objective behind this indirect method is to define a linear bounding function (or system) for the nonlinear system of interest. This linear function (or system) thereby provides an upper bound to the maximum responses of the nonlinear system. In addition, because this bounding system is linear, it can be used in a matched filter theory analysis with important benefits and guarantees. A description of each of these various components is a necessary first step towards understanding the present approach. These components include: 1). matched filter theory (MFT) for linear systems; 2). Volterra theory of nonlinear systems; and 3) zero-memory nonlinear elements (ZMNLEs). The process by which these various components are applied defines the present method which is described in a subsequent section.

\section{REVIEW OF METHODS}

\section{Matched Filter Theory (MFT) for Linear Systems}

As background for the remainder of this article, this section presents the key results from Ref. 3, the original development of MFT for computing maximized and timecorrelated gust loads for linear airplanes.

Figure 2 depicts the steps that must be employed to implement MFT and illustrates the intermediate and final products of the process. Frequency-response-function representations of atmospheric turbulence and airplane loads are combined in series and represent the "known dynamics" boxes in the figure. One-dimensional Gaussian atmospheric turbulence with the von Karman power spectrum is chosen. Load $y$ is the load to be maximized. Loads $z_{1}$ through $z_{\mathrm{n}}$ are the loads to be time-correlated with load $y$. There are three major steps in the process:

Step 1 is the application of an impulse function of unit strength to the combined linear system, producing the impulse response of load $y$.

Step 2 is the normalization of this impulse response by its own energy, followed by its reversal in time.

Step 3 is the application of this normalized reversed signal to the combined linear system, producing time histories of load $y$ and time histories of loads $z_{1}$ through $z_{n}$. Within the time history of load $y$, the maximum value is $y_{\text {max }}$.

For simplicity of discussion throughout this article, these three steps will be referred to as MFT. Within each step, significant results, interpretations, and implications are as follows:

Within step 1, the impulse response of atmospheric turbulence is shown as an intermediate quantity. The impulse responses of 
loads $z_{1}$ through $z_{n}$ can be computed but not used.

Within step 2, the normalized reversed signal, named the matched excitation waveform, now has unit energy. MFT guarantees that there is no other unit-energy excitation waveform that, when applied to the combined linear system, will produce a value of $y$ larger than $y_{\max }$. This guarantee is a fundamental result of MFT.

Within step 3, the response of atmospheric turbulence to the matched excitation waveform, named the critical gust profile, has a given level of probability. The responses of atmospheric turbulence to all other unit-energy excitation waveforms have the same given level of probability. Load $y$ is named the matched load, and loads $z_{I}$ through $z_{n}$ are time-correlated with load $y$. At each instant of time, the time history of the matched load is proportional to the autocorrelation function of load $y$ from a conventional random process analysis. At each instant of time, the time histories of loads $z_{l}$ through $z_{n}$ are proportional to their corresponding cross-correlation functions with load $y$.

For more detailed information about the application of MFT to the calculation of maximized and time-correlated gust loads, including background, theoretical development, numerical implementation, and example calculations, the reader is urged to consult Ref. 3 .

\section{Volterra Theory}

The Volterra theory was developed by Volterra in $1930^{7}$. The theory is based on functionals, or functions of other functions, and subsequently became a generalization of the linear convolution integral approach that is applied to linear time-invariant (LTI) systems. The theory formulates the response of a nonlinear, time-invariant system as an infinite sum of multidimensional convolution integrals of increasing order, with the first term in the series being the standard linear convolution integral. Each multidimensional convolution integral in the series has an associated kernel. The firstorder kernel is simply the linear unit impulse response of the system and the higher-order kernels are multi-dimensional impulse responses. This infinite sum of multidimensional convolution integrals is known as the Volterrra Series and it is well detined in both the time and frequency domains. To date, the Volterra theory has been applied primarily to nonlinear electrical and electronic systems.

Wiener ${ }^{8}$ contributed significantly to the Volterra theory and, as a result, the theory is currently referred to as the Volterra-Wiener theory of nonlinear systems. Reference 9 presents a kernel identification technique based on auto- and cross-correlation functions that can be applied to nonlinear, timevarying systems. The textbooks by Rugh ${ }^{11}$ and Schetzen " and the work by Boyd, Chua, and Desoer ${ }^{12}$ and several others ${ }^{13-18}$ provide detailed descriptions of the Volterra-Wiener theory and are highly recommended to the interested reader.

The basic premise of the Volterra-Wiener theory of nonlinear systems is that any continuously-differentiable (i.e., smooth) nonlinear system can be modeled as an infinite sum of multidimensional convolution integrals of increasing order. This infinite sum is known as the Volterra Series and it has the form

$$
\begin{aligned}
& \mathrm{y}(\mathrm{t})=\mathrm{h}_{0}+\int_{0}^{\infty} \mathrm{h}_{1}(\mathrm{t}-\tau) \mathrm{u}(\tau) \mathrm{d} \tau+ \\
& \int_{0}^{\infty} \int_{0}^{\infty} \mathrm{h}_{2}\left(\mathrm{t}-\tau_{1}, \mathrm{t}-\tau_{2}\right) \mathrm{u}\left(\tau_{1}\right) \mathrm{u}\left(\tau_{2}\right) \mathrm{d} \tau_{1} \mathrm{~d} \tau_{2}+\ldots \\
& +\int_{0}^{\infty} \ldots \int_{0}^{\infty} \mathrm{h}_{\mathrm{n}}\left(\mathrm{t}-\tau_{1}, \ldots, \mathrm{t}-\tau_{\mathrm{n}}\right) \mathrm{u}\left(\tau_{1}\right) \ldots \mathrm{u}(\mathrm{m}) \mathrm{d} \tau_{1} \ldots \mathrm{d} \tau_{\mathrm{n}}+\ldots
\end{aligned}
$$

where $y(t)$ is the response of the nonlinear system to $u(t)$, an arbitrary input; $h_{1}$ is the first-order kernel or the linear unit impulse response; $h_{2}$ is the second-order kernel, and $h_{n}$ is the nth order kernel.

Inspection of Equation (1) reveals some very interesting features that are characteristic of the Volterra series. If the kernels of order two and above are zero, then the response of the system is linear and is completely described by a linear unit impulse response $h(t)$ and the standard linear convolution integral.

The higher-order kernels $h_{n}$ are the responses of the nonlinear system to multiple unit impulses, with the number of impulses applied to the system equal to the order of the kernel of interest : e.g., $h_{2}$ is the 
response of the nonlinear system to two unit impulses applied at two varying points in time, $\tau_{1}$ and $\tau_{2}$. The mathematical definition follows directly for the $\mathrm{n}^{\text {th }}$ order kernels although visualization of these functions can become difficult for orders greater than three. The nonlinear kernels are measures of the relative influence of a particular nonlinear term of the series on the system's overall time response, which is a measure of nonlinearity. This is a temporal measure of nonlinearity and is referred to as memory. As a result, Volterra systems are referred to as nonlinear sytems with memory. The concept of memory is discussed in greater detail in the discussion of the ZMNLEs.

One approach for obtaining Volterra series representations of physical systems is to assume that the system is a 'weakly' nonlinear system. A system that is weakly nonlinear is a system that is well defined by the first few kernels of the Volterra series so that the kernels greater than second or third order fall off rapidly and are negligible. Boyd, Tang, and Chua ${ }^{19}$ mention some physical systems that are accurately modeled as weakly nonlinear systems including electromechanical and electroacoustic transducers and some biological systems. In the present study, it is assumed that the nonlinear , closed-loop aeroelastic system is a weakly nonlinear, second-order system. Although third-order kernels do need to be identified in order to verify this assumption, third-order analysis is beyond the scope of the present study. Results are therefore, limited to the identification of the secondorder kernel, or $h_{2}$, so that Equation (1) becomes

$\mathrm{y}(\mathrm{t})=\mathrm{h}_{0}+\int_{0}^{\infty} \mathrm{h}_{1}(\mathrm{t}-\tau) \mathrm{u}(\tau) \mathrm{d} \tau+$

$\int_{0}^{\infty} \int_{0}^{\infty} h_{2}\left(t-\tau_{1}, t-\tau_{2}\right) u\left(\tau_{1}\right) u\left(\tau_{2}\right) d \tau_{1} d \tau_{2}$

In the present study, these Volterra kernels are identified using the same unit pulse identification technique described in Refs. 20 and 21 .

Since the Volterra series is a generalization of the linear convolution concept, and since the linear MFT technique is based on the linear convolution concept, the Volterra series can be viewed as a framework for generalizing the MFT technique to nonlinear systems. This is discussed in a subsequent section of this paper.

\section{Zero-Memory Nonlinear Elements (ZMNLES)}

In order to determine the applicability of the Volterra theory to the present problem, the nonlinear system of Figure 2 needs to be classified with regard to the nature of its nonlinearity. The only source of nonlinearity is the nonlinear element (NLE) in Figure 2. It is well known that saturation and deadband functions are zero-memory (memoryless) functions ${ }^{22}$. A zero-memory function is a function with an instantaneous response (output). That is, the output of a zero-memory function depends only on the present value of the input, regardless of previous values of the input. An example of a linear zero-memory function is $y=5 x$ where the input is $x$ and the output is $y$. If a series of $x$ values are input to this function, the output due to one of those $x$ values is the same regardless of the order of the $x$ values. So for $x=2, y=10$ regardless of whether $x=2$ is the first $x$ value, the last $x$ value, or any $\mathrm{n}^{\text {th }}$ value in between. Therefore, the timecorrelation between input values is not important. An example of a nonlinear zeromemory function is $y=2 x^{2}$.

Simply stated, a system with memory is a system that continues to respond after the input has been removed (or set to zero). Therefore, a system with memory will exhibit an impulse response that is a function of time whereas a zero-memory system will exhibit an impulse response that is single-valued. Since convolution is the scaling and shifting of an impulse response (memory function), the output of a system with memory will be affected by the timecorrelation (relative ordering) of the input values. Linear and nonlinear systems can be classified as either zero-memory systems or as systems with memory. Typically, zeromemory systems are easier to analyze than systems with memory.

An important characteristic of zeromemory nonlinear functions is that they can be scaled linearly with the input. Scaling of 
a ZMNLE consists of two operations: scaling of the appropriate characteristic of the ZMNLE and scaling of the input function. For a saturation function, for example, the saturation level is referred to as the threshold value. So if a saturation function is defined with threshold values at \pm 1 , scaling of this function by a factor of two would consist of new threshold values at \pm 2 . Scaling of the input function by a factor of two then completes the scaling process. This process is depicted in Figures 3(a) and 3(b). This characteristic of ZMNLEs will be exploited to develop the present methodology.

In addition to being zero-memory functions, the ZMNLEs are also discontinuous (or piecewise continuous). Clearly these functions are piecewise linear but a nonlinear effect is introduced when input values switch back and forth between the piecewise linear functions. Formal application of the Volterra theory requires the smoothing of ZMNLEs so that the nonlinear system under investigation is continuously differentiable. This smoothing is accomplished via polynomial functions of the lowest order sufficient to capture the effects of the ZMNLE. The zero-memory nature of these elements reduces the polynomial smoothing process to a simple curve-fitting process. Polynomials of various orders are defined for each ZMNLE and compared with the original function to guarantee accuracy. An important point to be made is that the range of input amplitudes to each ZMNLE needs to be defined prior to any smoothing approximation. The range of input amplitudes (abcissa values) should exceed the maximum expected value in order to guarantee an accurate use of the curve fit.

\section{COMPUTATIONAL PROCEDURE}

The system of Fig. 1 is a nonlinear, closed-loop system consisting of a linear system with memory (aeroelastic vehicle) and a ZMNLE representing a simplified controller. The ZMNLE classifies the system as being nonlinear, but its zeromemory nature simplifies the nature of the nonlinearity and, therefore, simplifies the nature of the problem as well. Assuming an appropriate smoothing of the ZMNLE (a requirement for Volterra theory), two options exist for gust loads analysis of this system: 1). direct application of the MFT technique to nonlinear systems via the Volterra theory; or 2). an indirect approach that exploits the scalability of ZMNLEs.

A direct application of the MFT technique to a nonlinear system would be via the Volterra theory of nonlinear systems. Clearly, since the linear MFT technique relies on the mathematical advantages of the linear impulse response, a direct extension of the linear MFT to nonlinear systems is via the Volterra theory which relies on linear and nonlinear impulse responses. This direct application would involve the computation of an appropriate number of Volterra kernels of the system. The incremental contribution of the higher-order (nonlinear) kernels to the first-order autocorrelation function then can be computed, resulting in an autocorrelation function that includes nonlinear effects. The incremental contribution of the higher-order kernels asymptotically approaches a limit. This limit would be the maximized response including nonlinear terms. This direct approach would be a direct extension of the MFT to a nonlinear system. This direct approach, however, could impose an undesirable computational burden.

Alternatively, an indirect method is presented that circumvents the computational burdens of the direct approach mentioned above. This indirect method consists of three steps: 1). scaling of the original nonlinear system, 2). computation of the first-order (linearized) Volterra kernel of the scaled nonlinear system, and 3). application of MFT for linear systems using the kernel (impulse response) from step 2 . The basic strategy consists of computing the first-order kernel (linearized component) for the scaled nonlinear system and using this linearized component in a linear MFT sense to provide an upper bound to the original nonlinear system of interest. A roadmap for this indirect method is presented as Table 1, which will be used as a guide to aid the reader in understanding the method and the results presented in the next section. Reference to a step number corresponds to a step outlined in Table 1.

The nonlinear system of interest, hereby referred to as the nominal system (Fig. 1) is 
transformed into a continuously-differentiable nonlinear system by substituting the original discontinuous ZMNLE with a smoothedversion of the ZMNLE using a polynomial curve fit (step 1). This smoothed system (Fig. 4) is now suitable for analysis using Volterra theory and can be defined using Eq. (1). Truncation of the higher-order kernels for this system would yield the first-order (linearized) kernel (step 2). The first-order kernel of this system alone, however, cannot be used in a linear MFT analysis since the contribution of the higher-order kernels would be ignored. Recall that the guarantees of the MFT are limited to linear systems where the higher-order terms are, by definition, zero. A direct application of the nonlinear version of the MFT using Volterra theory (as described above) is certainly appropriate at this stage. The remaining discussion and results, however, are limited to the indirect method, the main focus of this paper. Application of the direct method is delayed to a future publication.

The next step in the indirect approach (step 3 ) consists of the scaling process (Figs. $3 a$ and $3 b$ ). Scaling of the nominal system (Fig. 1) is achieved by scaling the input (setting the gain at the input) and scaling the threshold values of the ZMNLE by the same amount. This scaled system (Fig. 5) is guaranteed to yield linearly-scaled responses of the nominal system. So that if the scale factor is greater than one, then for any given input, the response of the scaled system will always be greater than the response of the nominal system (by the scale factor). The new (scaled) ZMNLE is then replaced with a smoothed-version of the ZMNLE so that Volterra theory can be applied to the scaled system as well (step 4). Since the responses from the scaled system will always bound the responses from the nominal system, the scaled system is referred to as the bounding system (Fig. 6). This system can now be defined using Eq. (1) as well. It is important to mention that the kernels from step 2 for the smoothed, nominal system are different from the kernels of the smoothed, bounding systems from step 4 (Table 1).

An appropriate bounding system is detined by selecting a scale factor such that the first-order response (truncated Volterra model) of the bounding system (step 5) provides an upper bound to the full nonlinear response of the nominal system. This is facilitated by the use of square wave inputs which excite the entire frequency spectrum of each system. Comparisons are made between the full nonlinear response of the nominal system (step 2) and the first-order response of the bounding system (step 5). Once the bounding system is defined, its first-order impulse response can be used in a MFT analysis.

Application of the aforementioned technique to a simplified Airbus A310 aeroelastic model is presented in the next section.

\section{RESULTS}

The Airbus A310 linear mathematical model used in this study is a simplified version of the complete math model of the vehicle $^{6}$. This symmetric aeroelastic model consists of rigid body pitch and plunge degrees of freedom in addition to first wing bending $(1.9 \mathrm{~Hz})$, engine lateral $(2.3 \mathrm{~Hz})$, and engine pitch/inner wing torsion $(3.5 \mathrm{~Hz})$ degrees of freedom. All flexible modes are assumed to have a structural damping of $3 \%$. The rigid body degrees of freedom were removed to simplify the current analysis.

The output loads for this system include engine lateral acceleration ( $\mathrm{g}$ ), wing bending moment (lb-ft), wing torque (lb-ft), and aircraft c.g. normal acceleration (g). External inputs to the model include vertical gust velocity, spoiler, and aileron deflections. For the present analysis, the only input that is investigated is the vertical gust velocity input.

Steady state aerodynamics are assumed and lift growth and gust penetration effects are not modeled. The steady state aerodynamics are defined for the following flight condition:

$$
\begin{aligned}
\text { Altitude } & =25000 \mathrm{ft} . \\
\text { Mach } & =0.84 \\
\text { Veas } & =571.9 \mathrm{ft} / \mathrm{s}(\text { Vtas }=854.3 \mathrm{ft} / \mathrm{s}) \\
\text { Weight } & =290000 \mathrm{lb}
\end{aligned}
$$

The nonlinear closed-loop system is defined by the feedback of the normal acceleration signal through a nonlinear element. A block diagram of 
this mathematical model is presented as Figure 7. The ZMNLE for this example consists of an offset saturation element with thresholds of 0 and 10 degrees. An offset saturation function is a saturation function that is offset from the origin. This corresponds to simulation of a spoiler surface which can only be deflected in one direction. A more detailed description of this mathematical model can be found in Ref. 6 . In order to include a model of the gust system as part of the overall analysis, consistent with models used in previous MFT analyses, a von Karman pre-gust filter is added to the system of Figure 8. In addition, in order to scale the input appropriately, a gain block has been placed at the gust velocity input. The $\mathrm{A}$ and $\mathrm{B}$ state-space matrices were discretized for a time step of 0.01 and all analyses were performed in discrete time.

Results for validation of the polynomial smoothing process (Step 2) can be seen in Figure 8. Figure 8 is a comparison of the wing bending moment reponses due to a square wave input (1 $\mathrm{Hz}$, unit amplitude) for an offset saturation function (thresholds of 0 and 20) in its original discontinuous form and a smoothed version of the function. As can be seen, the responses are nearly identical. Although not presented here, wing bending moment reponses due to a $1 \mathrm{~Hz}$ square wave with double the amplitude (amplitude=2.0) for the discontinuous and smoothed offset saturation functions (same thresholds) showed excellent agreement as well.

The scaling process using a scale factor of two, applied to the ZMNLE of the system of Figure 7, was evaluated by applying a square wave input ( $1 \mathrm{~Hz}$, unit amplitude) via the gust velocity input and comparing the output wing bending moment responses. Presented in Figure 9 is a comparison of wing bending moment responses due to square wave inputs for an offset saturation NLE. One response is due to a square wave input ( $1 \mathrm{~Hz}$, unit amplitude) for offset saturation thresholds of 0 and 10 . The second response is due to a square wave input with double the amplitude (amplitude $=2.0$ ) and for offset saturation thresholds of 0 and 20 . It is clear that these responses are linearly related by a factor of two. The scaling process is therefore applicable to this complex system.

Once the smoothing and scaling have been performed and evaluated, Volterra kernel identification can be performed. As mentioned previously, the goal is to define a scaled, nonlinear system (bounding system) whose linear
Volterra truncation (first-order kernel) can be used to bound the full nonlinear response of the nominal system. In this case, the nominal system consists of the offset saturation with thresholds of 0 and 10 and unit maximum input amplitude. In order to define an appropriately scaled system, the first-order kernel of the bending moment response for the nominal system is generated and presented as Figure 10. The first-order kernel for the nominal system (step 2) is seen to be numerically well-behaved, exhibiting a decaying response.

The first-order kernel (Fig. 10) is convolved with a square wave input and compared to the full system response to the same square wave input. This comparison is presented as Figure 11 where it can be seen that the linear Volterra truncation (first-order kernel) captures the dominant phasing of the full nonlinear response while underpredicting the maximum peaks. This indicates that the scaled system needs to be scaled so that its first-order kernel response is larger than the response of the nominal (fully nonlinear) response. A scaling factor of two was chosen as a test value. This scale factor of two translates to an offset saturation with thresholds of 0 and 20 (double the original value of 10) and a maximum input amplitude of two (double the input gain).

The first-order kernel of the bounding function (linear Volterra truncation of scaled system, step 5) was then computed. This kernel was convolved with the square wave input used to generate the responses in Fig. 11. Shown in Figure 12 is a comparison of the response shown in Figure 11 with the added response due to the first-order response of the bounding system. It can be seen that the first-order response of the bounding system bounds the nonlinear response of the nominal system. More importantly, the first-order kernel of the bounding system can be used in a linear MFT analysis for determining maximized and time-correlated loads. All the guarantees and conveniences of the linear MFT can be applied to the bounding function (firstorder kernel of the bounding system). Therefore, the problem of predicting maximized gust loads for an apparently complex nonlinear system has been reduced to a simpler problem resulting in a linear bounding function that can be used to exploit powerful linear system techniques such as MFT. This was made possible by the proper classification of the nonlinear nature of the system of interest. 
An important issue remaining to be addressed, however, is determination of an optimal scaling factor " $\mathrm{A}$ " in Fig. 3b. In Figure 12, the scaling factor chosen resulted in peak loads of the bounding system that are on the order of $50 \%$ larger than the peak loads of the nominal system. Thus for this example problem, a scaling factor of 2.0 is obviously too large, potentially leading to an overdesigned wing structure. This is clearly an iterative process. Since the scaling is arbitrary, the scale factor could be reduced to a more appropriate level. Additional research is required to address this issue as well as issues regarding production implementation (industry environment), multiple nonlinearities, and direct application of MFT via Volterra kernel autocorrelations.

\section{CONCLUDING REMARKS}

A method has been presented that provides a linearized impulse response function that can be used to bound the nonlinear response of a nonlinear, closed-loop system for computation of maximized and time-correlated gust loads. The method is based on the Volterra theory of nonlinear systems and exploits fundamental properties of zero-memory nonlinear elements such as saturation and deadband functions. The method is computationally efficient and can provide guaranteed bounds on the maximum response of the nonlinear system. In addition, linear matched filter theory (MFT) can be applied to the linearized impulse response function directly. Although the foundation for the method has been defined, additional work is needed in developing a production-level process.

\section{REFERENCES}

'Barnes, T. J. "Harmonization of U.S. and European Gust Criteria for Transport Aircraft." Proceedings of the 17th Congress of the International Council of the Aeronautical Sciences, Stockholm, Sweden, Sept. 9-14, 1990. pp. 586-593.

2Graham, D., and McRuer, D., "Retrospective Essay on Nonlinearities in Aircraft Flight Control," Journal of Guidance, Control, and Dynamics, Vol. 14. No. 6, 1991, pp.1089-1099.
'Potolzky, A. S., Zeiler, T. A., and Perry, B., III, "Calculating Time-Correlated Gust Loads Using Matched Filter and Random Process Theories," Journal of Aircraft. Vol. 28, No. 5, 1991, pp 346-352.

${ }^{4} J o n e s$, J. G., Statistical-Discrete-Gust Method for Predicting Aircraft Loads and Dynamic Response," Journal of Aircraft, Vol. 26, No. 4, 1989, pp.382-392.

${ }^{5}$ Scott, R. C., Pototzky, A. S., and Perry, B., III, "Computation of Maximized Gust Loads for Nonlinear Aircraft Using Matched-Filter-Based Schemes," Journal of Aircraft, Vol. 30, No. 5, September-October 1993, pp. 763-768.

${ }^{6}$ Pfeil, A. L., An Investigation of a Proposed Airworthiness Requirement Based on the SDG Method: Aircraft Mathematical Model, SDL Report No. SDL-231-TR-1, Issue 1, June 1996.

${ }^{7}$ Volterra, V.: Theory of Functionals and of Integral and Integro-Differential Equations, Dover Publications, Inc., New York, 1959.

${ }^{8}$ Wiener, N.: "Response of a Nonlinear Device to Noise," dissertation No. 129, Radiation Laboratory, M.I.T., Cambridge, Massachusetts, April 1942. (Also published as U.S. Department of Commerce Publications PB-58087.)

${ }^{9}$ Lee, Y.W.; and Schetzen, M.: "Measurement of the Wiener Kernels of a Non-linear System by Crosscorrelation," International Journal of Control, Volume 2(3), pp. 237-254, September 1965.

${ }^{10}$ Rugh, Wilson J. : Nonlinear System Theory, The Volterra-Wiener Approach, The Johns Hopkins University Press, 1981.

"Schetzen, Martin : The Volterra and Wiener Theories of Nonlinear Systems. John Wiley \& Sons, 1980.

${ }^{12}$ Boyd, S. ; Chua, L. O. ; and Desoer, C. A. : Analytical Foundations of Volterra Series, IMA Journal of Mathematical Control \& Information, 1984, Volume 1, pp. 243-282.

1.3oyd, S.P.: "Volterra Series: Engineering Fundamentals," PhD Dissertation. University of California, Berkeley, 1985. 
${ }^{1-}$ Bedrosian, E.; and Rice, S. O.: "The Output Properties of Volterra Systems (Nonlinear Systems with Memory) Driven by Harmonic and Gaussian Inputs," Proceedings of the IEEE, Volume 59, Number 12, December 1971.

${ }^{15} \mathrm{Ku}, \mathrm{Y} . \mathrm{H}$.; and Wolf, A. A.: "Volterra-Wiener Functionals for the Analysis of Nonlinear Systems." Journal of the Franklin Institute, Volume 281, Number 1, January 1966.

${ }^{16}$ Brilliant, M. B.: "Theory of the Analysis of Nonlinear Systems," Technical dissertation 345, M.I.T. Research Laboratory of Electronics, March 1958.

${ }^{17}$ Boyd, S. P.; and Chua, L. O.: "Fading Memory and the Problem of Approximating Nonlinear Operators with Volterra Series" IEEE Transactions on Circuits and Systems, Volume CAS-32, Number 11, November 1985.

${ }^{18}$ Flake, R. H., "Volterra Series Representation of Nonlinear Systems," Transactions of the American Institute of Electrical Engineers, Volume 81, 1962, Part II: Applications and Industry.

${ }^{19}$ Boyd, Stephen ; Tang, Y. S. ; and Chua, Leon A. : "Measuring Volterra Kernels," IEEE Transactions on Circuits and Systems, Volume CAS-30, Number 8, August 1983.

${ }^{20}$ Silva, W. A., "Extension of a Nonlinear Systems Theory to General-Frequency Unsteady Transonic Aerodynamic Responses," AIAA Paper No. 93-1590, Presented at the 34th Structures, Structural Dynamics, and Materials Conference, La Jolla, California, April 19-21, 1993.

"Silva, W. A., "Discrete-Time Linear and Nonlinear Aerodynamic Impulse Responses for Efficient CFD Analyses," PhD Dissertation, College of William \& Mary, December 1997.

"'Bendat, J. S., Nonlinear System Analysis \& Identification from Random Data, A WileyInterscience Publication, 1990.

\begin{tabular}{|c|c|}
\hline $\begin{array}{l}\text { Nominal } \\
\text { Systems }\end{array}$ & $\begin{array}{l}\text { Bounding } \\
\text { Systems }\end{array}$ \\
\hline $\begin{array}{l}\text { Nominal } \\
\text { Figure } 1\end{array}$ & $\begin{array}{l}\text { Step } 3 \\
\text { Figure } 5\end{array}$ \\
\hline $\begin{array}{l}\text { Step } 1 \\
\text { Figure } 4 \\
\text { Eq. (1) }\end{array}$ & $\begin{array}{l}\text { Step } 4 \\
\text { Figure } 6 \\
\text { Eq. (1) }\end{array}$ \\
\hline $\begin{array}{l}\text { Step } 2 \\
\text { Eq. (1) } \\
\text { 1st-order } \\
\text { only }\end{array}$ & $\begin{array}{l}\text { Bounding } \\
\text { Eq. (1) } \\
\text { 1stt-order } \\
\text { only }\end{array}$ \\
\hline
\end{tabular}

Table 1 Roadmap for indirect method.

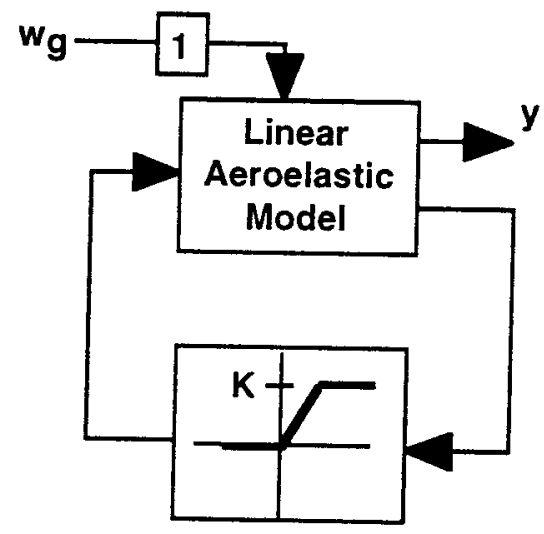

Nonlinear Element

Fig 1 Nonlinear, closed-loop (nominal) system

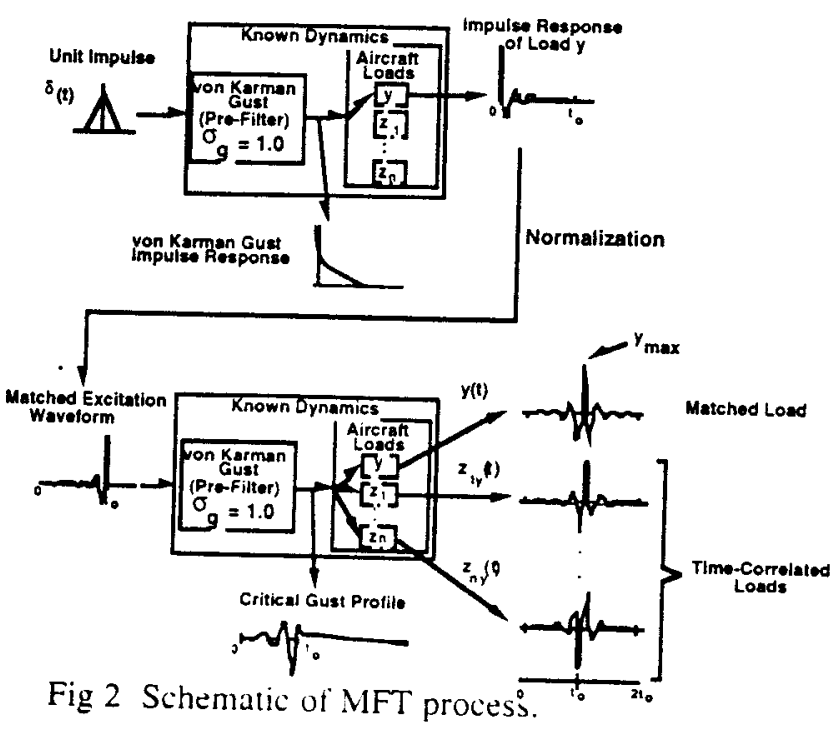




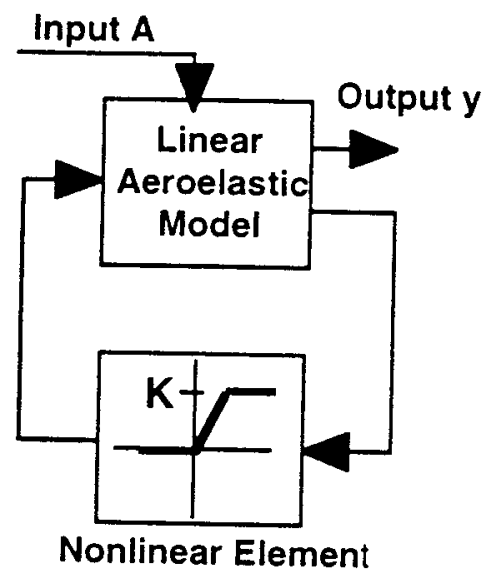

Fig 3a Nominal system

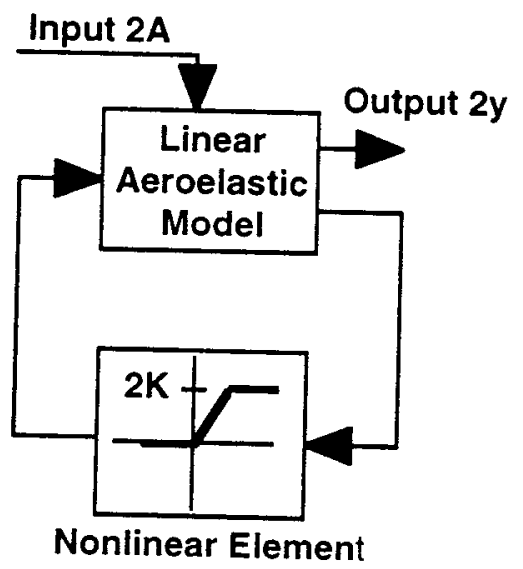

Fig 3b Scaled system

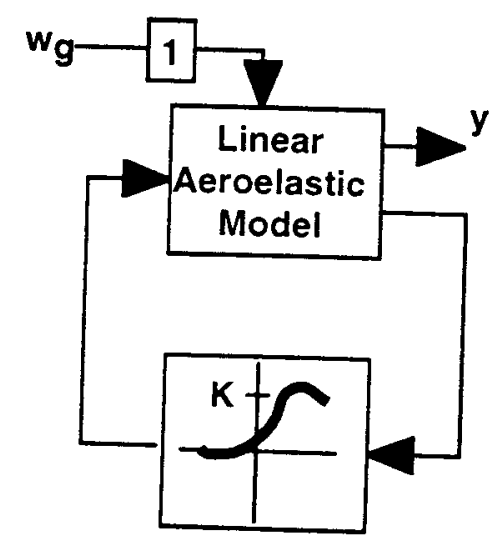

Smoothed Nonlinear Element

Fig 4 Smoothed nominal system

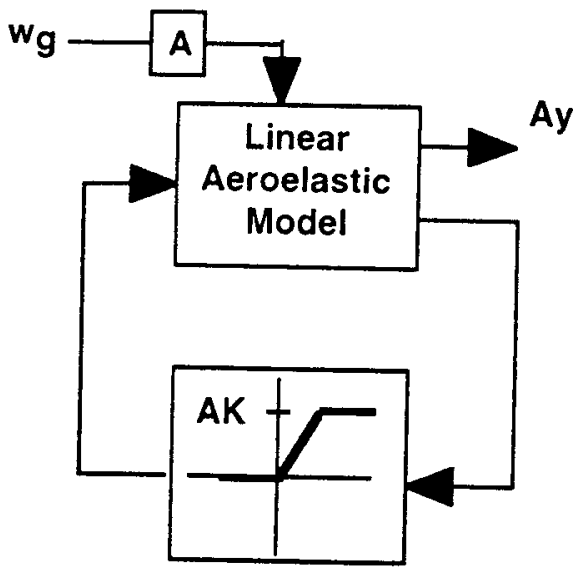

Nonlinear Element

Fig 5 Bounding (scaled) system

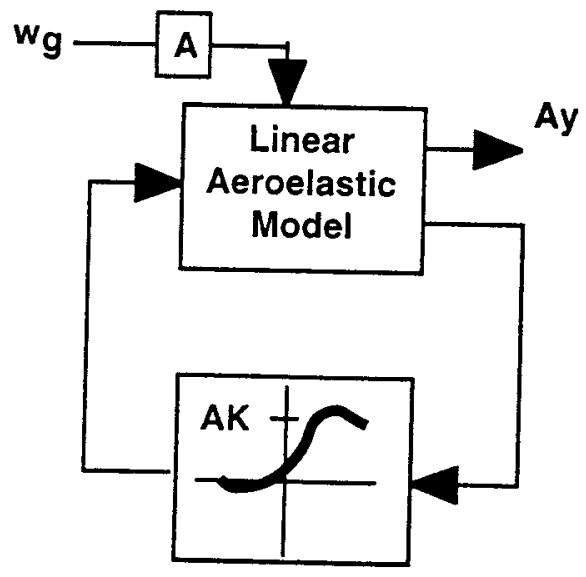

Smoothed Nonlinear Element

Fig 6 Smoothed bounding (scaled) system

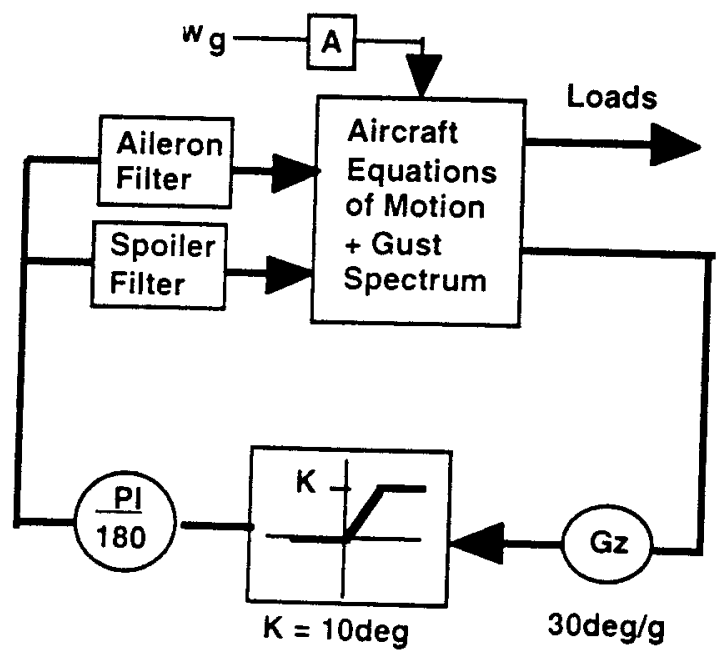

Fig 7 Simplified aeroelastic model of Airbus A310. 


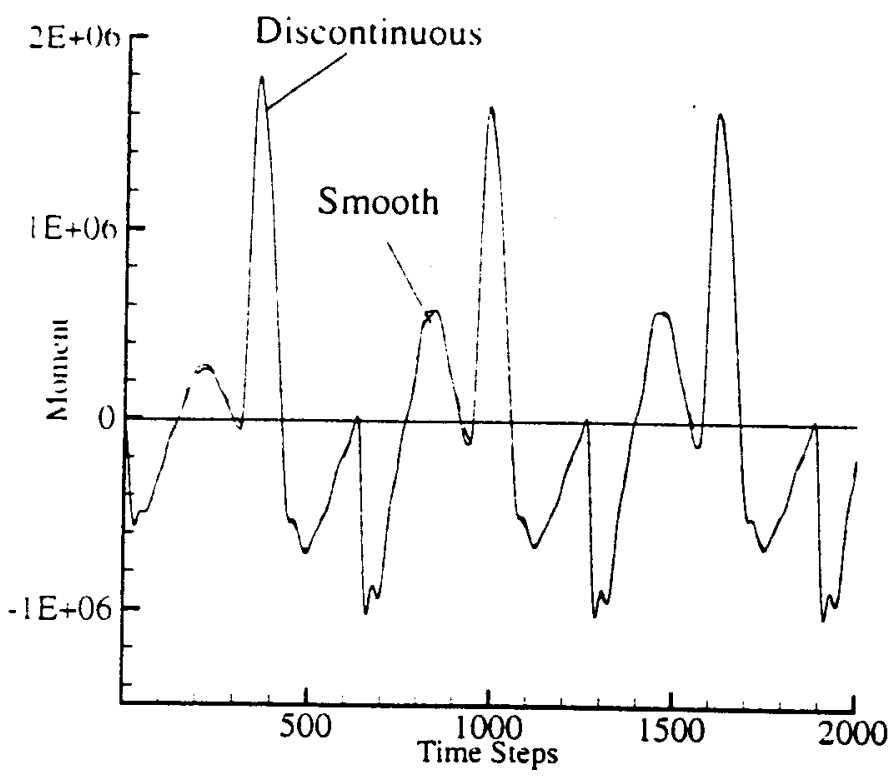

Fig 8 Comparison of discontinuous and smooth versions of the nominal system.

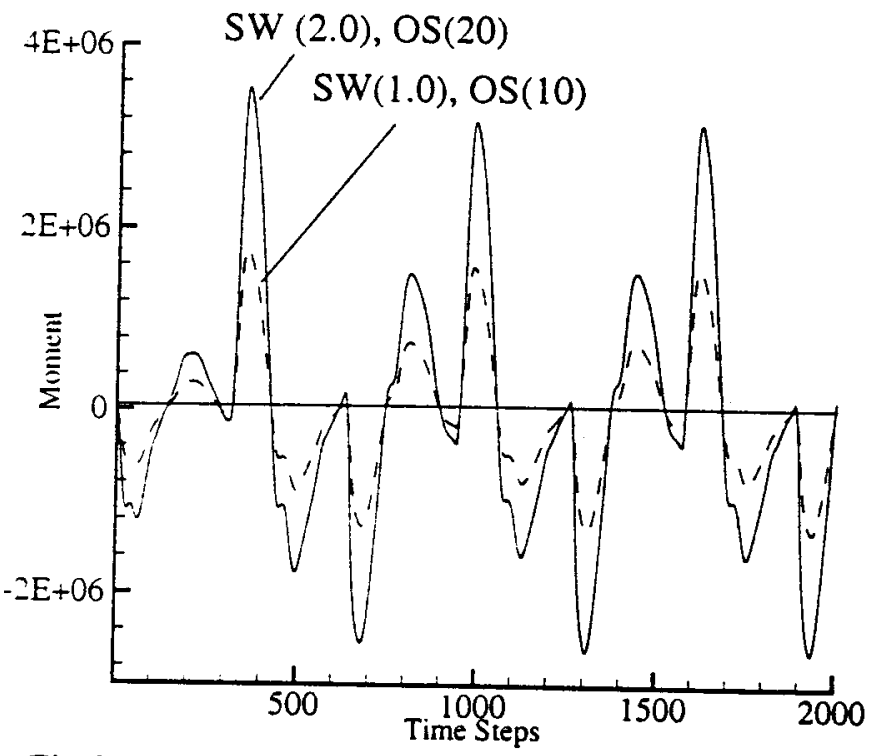

Fig 9 Effect of scaling using a square wave (SW) and offset saturation (OS) function.

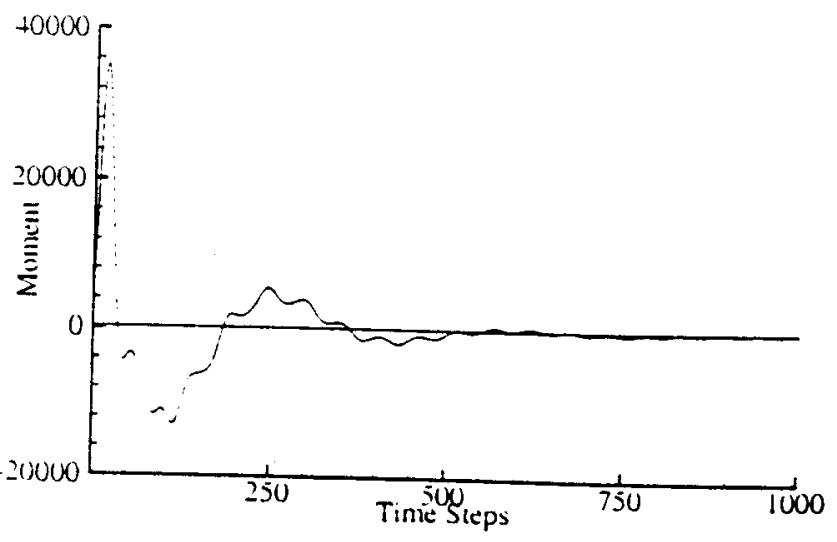

Fig 10 Linearized (first-order) impulse response for smooth, nominal system.

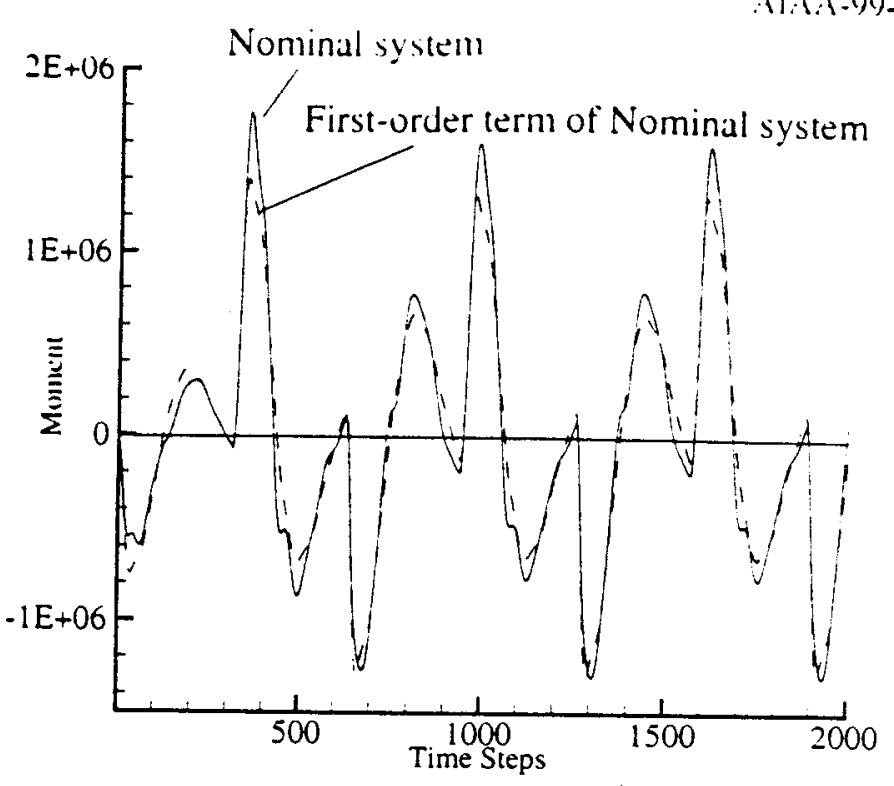

Fig 11 Comparison of full nonlinear response from nominal system and the first-order term of nominal system.

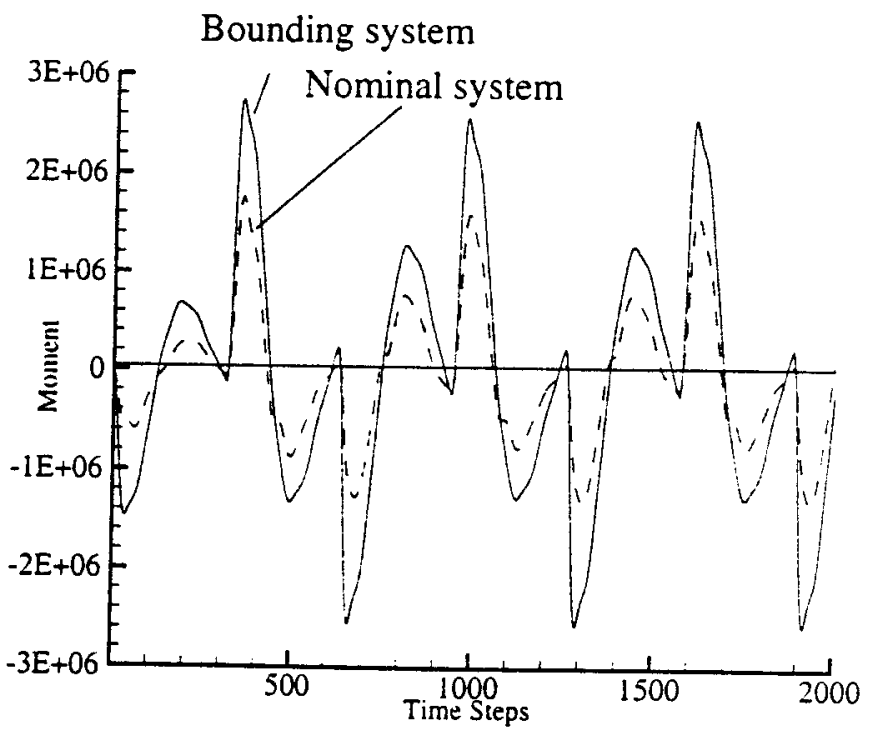

Fig 12 First-order term of bounding system used as an upper bound on the full (nonlinear) response of nominal system. 